Original Article

Journal of Epilepsy Research pISSN 2233-6249 / elSSN 2233-6257

Received November 16, 2020

Revised April 21, 2021

Accepted April 24, 2021

Corresponding author:

Jagarlapudi MK Murthy, MBBS, MD, DM

Department of Neurology, CARE Institute of

Neurosciences, CARE Hospitals, Road

No.10, Banjara Hills, Hyderabad 500 034,

India

Tel. $+91-04061656565$

Fax. +91-040-23320886

E-mail; jmkmurthy49@gmail.com

\section{Posterior Reversible Encephalopathy Syndrome Is the Common Cause of New-Onset Seizures in the Peripartum Period: A Tertiary Hospital-Based Study in South India}

\author{
Jagarlapudi MK Murthy, MBBS, MD, DM, Shyam K Jaiswal, MBBS, MD, DNB, \\ Keshava Anand Gaade, MBBS, DNB \\ Department of Neurology, CARE Institute of Neurosciences, CARE Hospitals, Hyderabad, India
}

\begin{abstract}
Background and Purpose: To study the aetiolgic spectrum of new-onset seizures in the peripartum period in south India.

Methods: This is a retrospective analysis of case records of women with new-onset seizures in the peripartum period admitted between 2005 and 2018 (13 years).

Results: Of the 41 women (mean age, 26.20 years; range, 19-35 years) admitted, 20 patients (48.7\%) had hypertensive disorders of pregnancy (HDP). Generalized tonic-clonic seizure $(88 \%)$ was the common seizure type. The aetiologies of new-onset seizures were: 1) pregnancy-related aetiologies in $33(80.5 \%)$ and 2$)$ non-pregnancy-related aetiologies in eight (19.5\%). Of the pregnancy-related aetiologies, posterior reversible encephalopathy syndrome (PRES) was the commonest cause in 24 patients $(58.5 \%)$. Seizure cluster presentation was common in patients with PRES $(p=0.0087)$. Of the eight women with non-pregnancy-related aetiologies, endemic central nervous system (CNS) infections accounted for three ( $7.3 \%$; brain tuberculoma in one and neurocystocercosis in two) of the aetiology. All the women had Glasgow outcome scale-5 outcome.
\end{abstract}

Conclusions: PRES was the common cause of new-onset seizures in peripartum period in this cohort. Endemic infections of CNS accounted for $7.3 \%$ of the total aetiological spectrum. This study suggests that the possibility of PRES should be considerd in woman with HDP and seizure cluster in peripartum period. (2021;11:49-55)

Key words: Peripartum period, New-onset seizure, Hypertensive disorders of pregnancy, Posterior reversible leukoencephalopathy syndrome, Cerebral venous thrombosis

\section{Introduction}

Seizures in the peripartum period involve less than $1 \%$ of pregnancies and are associated with significant morbidity and mortality to both the pregnant women and their unborn baby. ${ }^{1,2}$ Aetiologies of new-onset seizures in the peripartum period can be grouped into: 1) pregnancy-related and 2) non-pregnancy-related. ${ }^{1-3}$ The important pregnancy-related aetiologies include eclampsia, intracerebral haemorrhage, cerebral venous thrombosis (CVT), reversible cerebral vasoconstriction syndrome, posterior reversible encephalopathy syndrome (PRES), and thrombotic thrombocytopenic purpura. ${ }^{1-3}$ The aim of the study is to find any differences in the aetiological spectrum of new-onset seizures in peripartum period in this part of the world when compared to the aetiological spectrum seen in developed countries.

\section{Methods}

This is a retrospective study of case records of consecutive pregnant women with new-onset seizures during peripartum period, admitted to the neurological intensive care unit (NICU) in a tertiary-care corporate hospital in south India between January 2005 and December 2018 (13 years). Data collected included: age; period at presentation (antepartum or postpartum); interval between delivery and seizure(s) onset in days; presenting clinical symptoms; seizure type (single seizure and seizure clusters) and presentation (convulsive status epilepticus [CSE] and non-CSE [NCSE]); neurologic findings; blood pressure (BP) at admission and BP readings during the hospital stay; possible cause 
of seizure disorder; findings on magnetic resonance imaging (MRI) and MR-venography; electroencephalogram (EEG) findings; other relevant investigations; treatment received; and the outcomes. The criteria proposed by the International League against Epilepsy (ILAE) were used to categorize seizures into acute symptomatic seizures and unprovoked seizures. ${ }^{4}$ Seizures were classified using ILAE 2017 seizure classification. ${ }^{5}$ Diagnosis of generalized CSE (GCSE) was based on the criteria proposed by ILAE. ${ }^{6}$ Salzburg criteria were considered for the diagnosis NCSE. ${ }^{7}$ Seizure cluster was defined as 3 or more seizures within a 24 -hour period with a return to baseline between events. ${ }^{8}$ Outcome at three months was assessed using Glasgow outcome scale (GOS). ${ }^{9}$

\section{Operational definitions}

1. Peripartum period is defined as the period shortly before, during, and immediately after child birth. It includes antepartum and post partum periods. Postpartum period includes three phases: acute phase (6-12 hours), second phase (2-6 weeks), and third phase (up to 6 months). ${ }^{10}$

2. Hypertension in pregnancy is defined as systolic blood pressure (sBP) $>140 \mathrm{mmHg}$ and/or diastolic blood pressure (dBP) $>90 \mathrm{mmHg}$, taken at least 15 minutes apart, using the same arm. ${ }^{11}$

3. Chronic hypertension was defined as "hypertension that was present either pre-pregnancy or that developed at $<20$ weeks of gestation." ${ }^{11}$

4. Preeclampsia was defined as "a multisystem disorder of mid-to-late pregnancy diagnosed by the presence of de novohypertension after 20 weeks gestation accompanied by proteinuria ( $>300$ $\mathrm{mg}$ protein/24 hours urine) or evidence of maternal acute kidney injury, liver dysfunction, neurologic changes (encephalopathy and seizures), haemolysis or thrombocytopenia, or foetal growth restriction." Preeclampsia may occur intrapartum and less frequently early postpartum usually within 48 hours. Eclampsia was defined as "the development of seizures in a patient with preeclampsia." ${ }^{12}$

5. Gestational hypertension was defined as "hypertension that de- velops for the first time at more than or equal to 20 weeks gestation." ${ }^{11}$

6. Postpartum hypertension (PPHT) was "defined as hypertension appearing after delivery through 6 weeks postpartum. ${ }^{13}$ PPHT was considered a de novo condition when it followed a normotensive pregnancy. ${ }^{14}$

7. PRES is defined as a clinico-radiological syndrome that is typically characterized by headache, visual disturbances, and seizures associated with cortical and subcortical reversible vasogenic oedema in neuroimaging. ${ }^{15,16}$

\section{Stastical analysis}

Chi-square test was used as test of significance for categorical data. Unpaired T test was used as test of significance continuous data. $p$-value less than 0.05 was considered as statistically significance. Data was analysed using SPSS ver. 21 software (IBM Corp, Armonk, NY, USA).

\section{Results}

During the study period (13 years), 41 (mean age \pm standard deviation, $26.20 \pm 4.22$ years; range, 19-35 years) women were admitted to NICU for new-onset seizures during peripartum period, 12 (29\%) in the antepartum period and $29(71 \%)$ in the postpartum period. Of the 41 women with new-onset seizures, 20 (48.7\%) had HDP: eclampsia in four; gestational hypertension in three; and de novo PPHT in 13 (Table 1). Presenting clinical features in the total cohort were: seizures in 41 patients (100\%); headache in $15(36.6 \%)$; nausea and vomiting in six (12\%); blurring of vision or diminished vision in five (12\%); and neurological deficits in four $(9.7 \%)$ (Tables 1, 2). Seizure type by onset was: generalized tonic-clonic seizures (GTCS) in 36 patients (88\%); focal onset with impaired awareness motor to bilateral tonic-clonic seizures in three (7\%); and unknown onset motor seizures to bilateral tonic-clonic seizure in two $(5 \%) .^{5}$ At presentation 16 patients (39\%) had an isolated seizure, $22(54 \%)$ had

Table 1. Women with new-onset seizures during peripartum period: demographics

\begin{tabular}{lll}
\hline & \multicolumn{1}{c}{ Women with HDP $(n=20)$} & \multicolumn{1}{c}{ Women: normotensive $(n=21)$} \\
\hline Age, mean \pm SD (years) & $24.7 \pm 3.5$ (range, 19-31) & $27.62 \pm 4.43$ (range, 20-35) \\
Antepartum & Four women, 5-8 months amenorrhea & Seven women, 3.5-9 months amenorrhea \\
Postpartum & Sixteen women, days after delivery (mean days $\pm S D$, & Thirteen women, days after delivery (mean days $\pm S D$, \\
& $6.43 \pm 4.24$ days; range, 1-17 days) & $7.08 \pm 8.13$ days; range, 1-30 days) \\
Gravida status & G1:10; G2:5; G3:3; G4:2 & G1:10; G2:4; G3:4; G4:3 \\
\hline
\end{tabular}

HDP, hypertensive disorders of pregnancy; SD, standard deviation. 
seizure clusters, two (5\%) had GCSE, and one (2\%) had NCSE. Seizure cluster presentation was significantly more common in pa- tients with PRES when compared to patients with seizures due to other aetiologies ( $p=0.0087$ ). Patients with new-onset seizures due

Table 2. Clinical characteristics with PRES and other aetiologies

\begin{tabular}{lc}
\hline Clinical feature & Value $(n=41)$ \\
\hline Presenting symptoms in women with PRES & $24(58.5)$ \\
Seizures & $24(100.0)$ \\
Headache & $10(41.6)$ \\
Nausea and vomiting & $4(16.6)$ \\
Visual disturbances & $4(16.6)$ \\
Presenting symptoms in women with other aetiologies & $17(41.5)$ \\
Seizures & $17(100.0)$ \\
Headache & $5(29.0)$ \\
Nausea and vomiting & $2(12.0)$ \\
Visual disturbances & $1(6.0)$ \\
Neurologic deficits & $2(12.0)$ \\
\hline
\end{tabular}

Values are presented as number (\%).

PRES, posterior reversible encephalopathy syndrome.

Table 3. Seizure type by onset - ILAE 2017 seizure classification and presentation

\begin{tabular}{lccc}
\hline & Women with PRES $(n=24)$ & Women with other aetiologies $(n=17)$ & $p$-value \\
\hline Focal onset & $1(4.0)$ & $3(18.0)$ \\
Generalized onset & $22(92.0)$ & $12(70.0)$ \\
Unknown onset & $1(4.0)$ & $2(12.0)$ & $11(65.0)$ \\
Isolated seizure & $5(21.0)$ & $5(29.0)$ & 0.0050 \\
Seizure clusters & $17(71.0)$ & 1 & 0.0087 \\
Convulsive status epilepticus & $1(4.0)$ & $0(0.0)$ & \\
Non-convulsive status epilepticus & $1(4.0)$ & & \\
\hline
\end{tabular}

Values are presented as number (\%).

ILAE, International League against Epilepsy; PRES, posterior reversible encephalopathy syndrome.
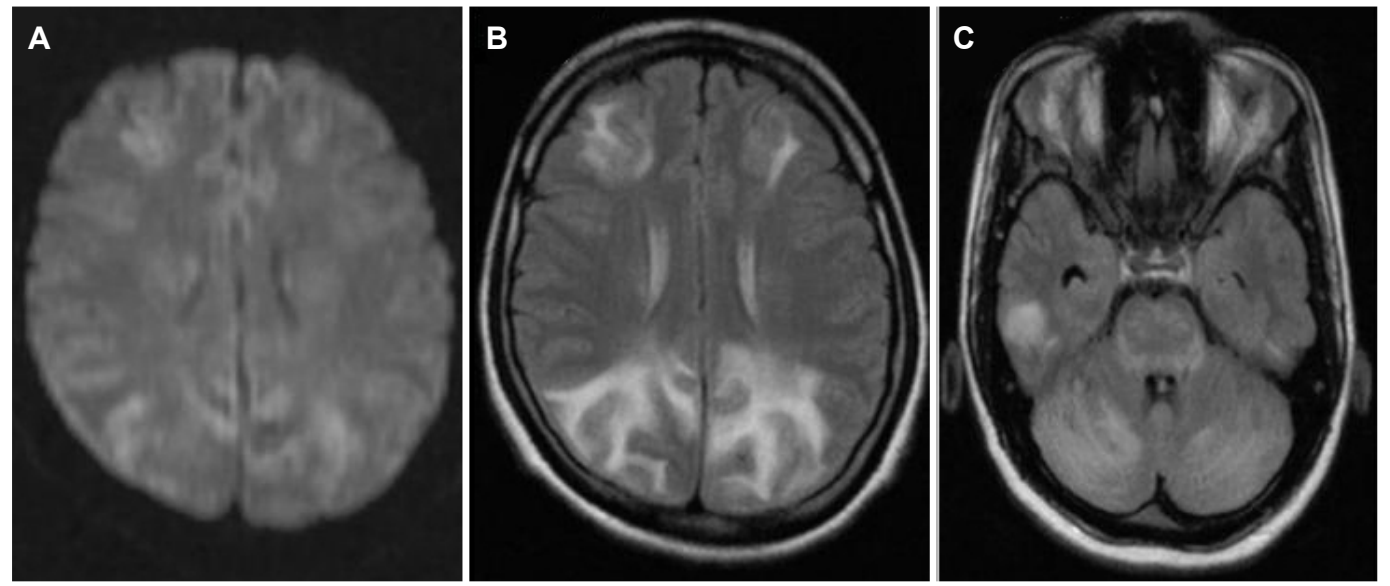

Figure 1. Magnetic resonance imaging in a normotensive patient with posterior reversible encephalopathy syndrome. (A) Diffusion-weighted imaging-iso images showing hypointensity in the subcortical location which shows bright signal on apparent diffusion coefficient (B) suggestive of increased diffusion; (C) fluid attenuated inversion recovery images showing cortical and subcortical hyperintensities in both occipital lobes. 

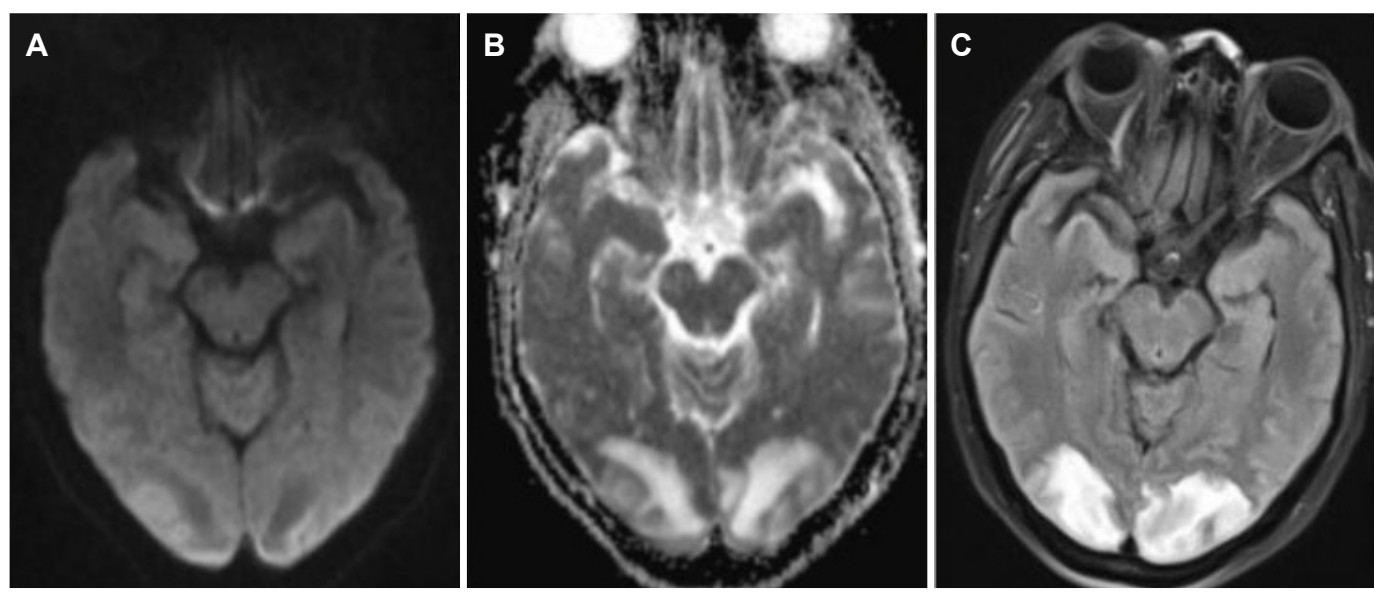

Figure 2. (A) Diffusion weighted imaging-iso images showing hypointensity in the subcortical location which shows bright signal on apparent diffusion coefficient (B) suggestive of increased diffusion; (C) fluid attenuated inversion recovery images showing cortical and subcortical hyperintensities in both occipital lobes.
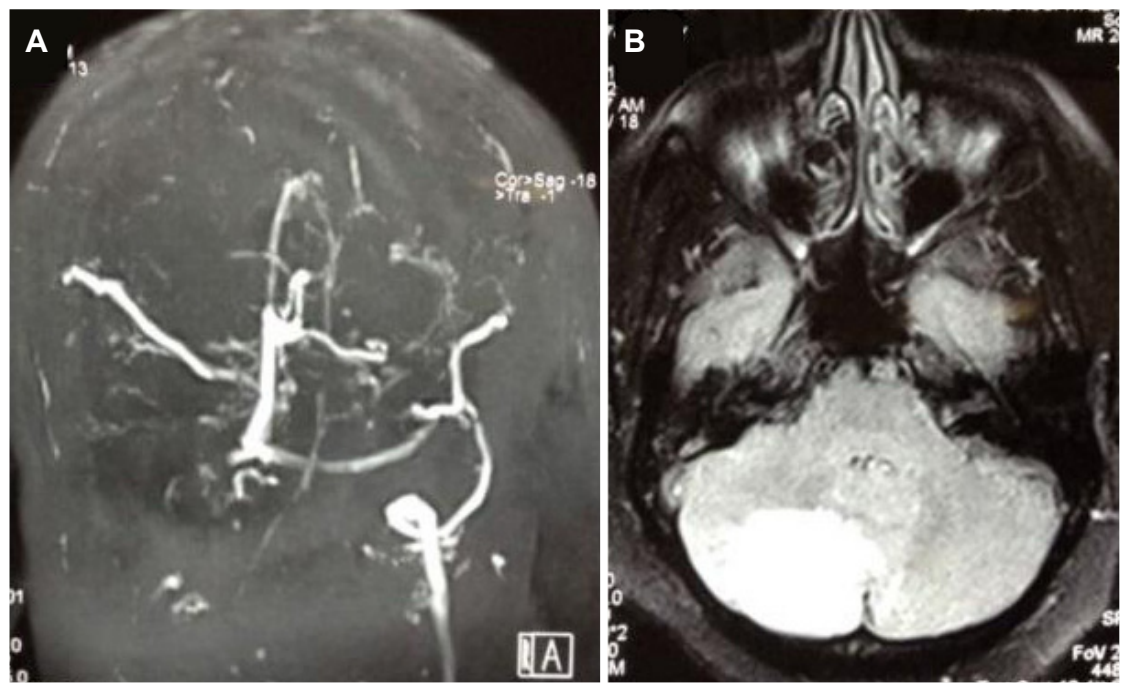

Figure 3. Magnetic resonance imaging (MRI) in a patient with CVT. (A) Non contrast MR cerebral venogram images showing lack of flow signal in the superior sagittal sinus, right transverse and sigmoid sinuses suggestive of occlusion. (B) Fluid attenuated inversion recovery images axial images at the level of middle cerebellar peduncles showing an area of swelling and edema in the medial and posterior aspects of right cerebellar.

to other aetiologies presented with an isolated seizure when compared to patients with PRES ( $p=0.005$ ) (Table 3). PRES was the cause in one patient each with GCSE and NCSE (Table 3).

EEG was normal in 33 patients (80.5\%); non-specific slow rhythms mostly in the posterior head regions were seen in four records, focal epileptiform discharges in two, and generalized spike-wave $>3.5 \mathrm{~Hz}$ interictal epileptiform discharges in one. One patient had continuous epileptiform discharges lasting for more than 10 seconds $(>2.5 \mathrm{~Hz})$ meeting the Sakzberg criteria for NCSE. ${ }^{7}$ In the patients with GCSE, continuous EEG monitoring was not done and EEGs were done after the remission of SE. The aetiologies spectrum was: 1) pregnancy-related in $33(80.5 \%)$ and 2) non-pregnancy-related in eight (19.5\%). PRES accounted for $58.5 \%$ of the total cohort and $72.7 \%$ of the pregnenancy-related aetiologies (Figs. 1, 2). CVT accounted for $15 \%$ of the pregnancy-related aetiologies and $12.2 \%$ of the total cohort (Fig. 3). Of the eight patients (19.5\%) with non-pregnancy-related aetiologies, three patients (37.5\%) had central nervous system (CNS) infections (tuberculosis and neurocysticercosis). In one patient the diagnosis of juvenile myoclonic epilepsy (JME) was made after the review of history and EEG features. One patient had unprovoked seizures due 
Table 4. New-onset seizures in peripartum: aetiology

\begin{tabular}{lc}
\hline & Value $(n=41)$ \\
\hline Pregnancy-related seizure disorder & $33(80.5)$ \\
Eclampsia & 2 \\
Posterior reversible encephalopathy syndrome & 24 \\
Includes two patients with eclampsia & \\
PRES in women with HDP & $18(75.0)$ \\
PRES in normotensive women & $6(25.0)$ \\
Reversible cerebral vasoconstriction syndrome & 2 \\
Cerebral venous thrombosis & 5 \\
Nonpregnancy-related aetiologies & $8(19.5)$ \\
Juvenile myoclonic epilepsy & 1 \\
Epilepsy of unknown aetiology & 1 \\
Epilepsy due to gliotic lesion & 1 \\
Neurocysticercosis & 2 \\
Tuberculoma & 1 \\
Hyponatremia & 2 \\
\hline
\end{tabular}

Values are presented as number (\%).

PRES, posterior reversible encephalopathy syndrome; HDP, hypertensive disorders of pregnancy.

to a gliotic scar and one patient had unprovoked seizure due to unknown cause (Table 4). ${ }^{4}$

The distribution of MRI-T2, diffusion-weighted imaging (DWI) and fluid attenuated inversion recovery (FLAIR) lesions, was parieto-occipital in 36 patients (87.7\%). This pattern of distribution was bilateral and symmetrical or asymmetrical (Fig. 1A, B). Four patients $(9.7 \%)$ had bilateral frontal lesions in addition to parieto-occipital lesions. One patient had extensive lesions involving parieto-occipital, frontal, temporal, cerebellum and brainstem (Fig. 2). There was no difference in the distribution and intensigty of the lesions between PRES in patients with HDP and PRES in normotensive patients.

\section{PRES and new-onset seizures}

PRES (24/41; 58.5\%) was the common aetiology in the total cohort and in patients with pregnancy-related aetiologies (24/33; $72.7 \%$ ). The mean age was $25.67 \pm 4.3$ years (19 years and 34 years). Five women presented in the antepartum period and 19 in the postpartum period. Of the 24 patients with PRES, 18 (75\%) had HDP and six (25\%) were nonhypertensive. None of the six normotensive patients had BP readings $\mathrm{sBP}>140 \mathrm{mmHg}$ and/or $\mathrm{dBP}>90 \mathrm{mmHg}$ during the hospital stay. The gravida status was either primigravida or gravida-2 (75\%). There were no significant differences in the clininical feature between PRES and non-PRES group. Seizure cluster as a presenting feature was significantly higher in patients with PRES when compared to patient with seizures due to other causes $(p=0.0087)$. There were no signigicant differences in the distribution and location of PRES lesions in the MRI between hypertensive and nonhypertensive patients. All patients had good functional recovery (GOS-5). None of the patient developed epilepsy during the follow-up.

\section{Treatment and outcome}

All the four women with eclampsia with and without PRES were treated with magnesium sulphate. Patients with gestational hypertension and PPHTN and PRES were treated with intravenous benzodiazepines (lorazepam or midazolam). In addition, patients with seizure cluster received intravenous loading dose of valproate/levetiracetam and short term (1-2 months) antiseizure medicatin (ASM) prophylaxis. Patients with GCSE and NCSE were treated as for the guidelines. Patients with specific causes received the appropriate medication. BP was treated as for the best practices of management of $B P$ in pregnant women. Of the total cohort, the outcome was GOS-5 in 39 patient (95\%) at 3 months and two women with CVT had GOS-4. All patients with PRES had GOS-5 outcome.

\section{Discussion}

The findings of the study were: 1) PRES (58.5\%) was the common cause of new-onset seizures in the peripartum period; 2) of the 24 patients with PRES, 18 (75\%) had HDP and $13(72.2 \%)$ had de novo PPHT and all the 13 patients with PPHT had PRES; 3 ) seizure cluster presentation was common in patients with PRES $(p=0.0087) ; 4)$ PRES was the pathological substrate in women with HDP and seizure cluster in the peripartum period; and 5) there were some differences in the aetiological spectrum in this cohort when compared to the spectrum seen in developed countries. Endemic infections of CNS accounted for $7.3 \%$ of the total cohort. CVT accounted for $12.2 \%$ of aetiology. In a large cohort of 428 patients with CVT from India, pregnancy-related CVT accounted for $9.8 \%$ of risk factors, ${ }^{17}$ whereas in the International Study on Cerebral Venous and Dural Sinus Thrombosis (ISCVT), 3-6\% were related to pregnancy. ${ }^{18}$ Among the most common causes of seizures in the peripartunm pereiod, eclampsia and pre-eclampsia were under represented in this study. This under representation of these two conditions may be explained as it was a neurological intensive care unit-based study.

Seizures are very common in patients with PRES and the reported 
frequency ranged between $74 \%$ and $87 \%{ }^{16,19}$ In women PRES occurs mostly in the setting of preeclampsia or eclampsia. ${ }^{20,21}$ In a retrospective study, PRES was found in more than $90 \%$ of eclamptic women and about $20 \%$ of preeclamptic women with neurological symptoms. ${ }^{22}$ In this study, of the 24 patients with PRES, 18 (75\%) had HDP and 13 (86.6\% \%) had de novo PPHTN. PPHTN is relatively common and can present either as a de novo condition following a normotensive pregnancy as in this study or as persistent hypertension following a pregnancy complicated by severe preeclampsia. ${ }^{14,23}$ The reported prevalence of HDP is high in developing countries. ${ }^{24}$ PPHTN following normotensive pregnancy may herald the development of seizures. ${ }^{24}$ Of the six normotensive women with PRES, five women presented in the postpartum period and none of them presented the BP recordings were SBP $>140 \mathrm{mmHg}$ and/or dBP $>90 \mathrm{mmHg}$ during the hospital stay. PRES occurring in normotensive pregnant women is rarely reported, mostly confining to single case reports. It is possible that these women had relative hypertension compared with the patient's baseline BP during peripartum period. Other possibility is that these women might have had transient hypertension due to pain, use of non-steroidal anti-inflammatory drugs (NSAIDs), and labour-related stress. ${ }^{25}$ None of the six patients had any predisposing risk factors for PRES.

A small study comparing the clinical features in women with HDP and PRES and women with PRES due to other predisposing disorders found no differences in the clinical features, ${ }^{26}$ whereas a large study has documented some differences in MRI features in women with HDP and PRES when compared to patients with PRES due to other causes and included less sever oedema, less cytotoxic edema, haemorrhage, contrast enhancement and more complete resolution. ${ }^{27}$ In our study there was no comparator to study the difference if any. However, we found no differences in the clinical and MRI features between patients with HDP and PRES and normotensive patients with PRES. In our study the sample size is small in the normotensive group for any reasonable conclusions. Except for eclampsia and pre-eclampsia, there are no evidence-based guidelines for treating seizures in patient with PRES due to other causes in peripartum period. Our protocol of treating new-onset seizures in patients with PRES due to other aetiologies is similar to the guidelines followed to treat any acute seizures. In this study 39 women (95\%) had GOS-5 outcome at 3-month follow-up. Two patients with CVT had GOS-4 outcome. PRES syndrome has an overall good short and long-term prognosis. Recurrence of PRES is rare. In patients with PRES, development of late epilepsy during long-term is infrequent. ${ }^{22}$

The major limitation of the study is small sample size. The study samples include very few cases of eclampsia. This may be related to the fact that our hospital had no active obstetric services till recently and the study was neurological intensive care unit-based study. All women were referred from other facilities. The strength of the study is that it characterizes the new-onset seizures in women in the peripartum period in this part of the world.

\section{Acknowledgements}

This study has the approval of the Institutional Ethics Committee (Institutional Ethics Committee meeting dated September 17, 2020, Item-13)

\section{Conflict of Interest}

The authors declare that they have no conflicts of interest.

\section{References}

1. Edlow $A G$, Edlow BL, Edlow JA. Diagnosis of acute neurologic emergencies in pregnant and postpartum women. Emerg Med Clin North Am 2016;34:943-65.

2. Edlow JA, Caplan LR, O'Brien K, Tibbles CD. Diagnosis of acute neurological emergencies in pregnant and post-partum women. Lancet Neurol 2013; 12:175-85.

3. Aya AG, Ondze B, Ripart J, Cuvillon P. Seizures in the peripartum period: epidemiology, diagnosis and management. Anaesth Crit Care Pain Med 2016;35 Suppl 1:S13-21.

4. Beghi E, Carpio A, Forsgren $L$, et al. Recommendation for a definition of acute symptomatic seizure. Epilepsia 2010;51:671-5.

5. Fisher RS, Cross JH, French JA, et al. Operational classification of seizure types by the international league against epilepsy: position paper of the ILAE commission for classification and terminology. Epilepsia 2017; 58:522-30.

6. Trinka $E$, Cock $H$, Hesdorffer $D$, et al. A definition and classification of status epilepticus--report of the ILAE task force on classification of status epilepticus. Epilepsia 2015;56:1515-23.

7. Leitinger $M$, Beniczky $S$, Rohracher $A$, et al. Salzburg consensus criteria for non-convulsive status epilepticus--approach to clinical application. Epilepsy Behav 2015;49:158-63.

8. Haut SR. Seizure clustering. Epilepsy Behav 2006;8:50-5.

9. Jennett $B$, Bond M. Assessment of outcome after severe brain damage. Lancet 1975;1:480-4.

10. Romano M, Cacciatore A, Giordano R, La Rosa B. Postpartum period: three distinct but continuous phases. J Prenat Med 2010;4:22-5.

11. Magee LA, Pels A, Helewa M, Rey E, von Dadelszen P; Canadian Hypertensive Disorders of Pregnancy (HDP) Working Group. Diagnosis, evaluation, and management of the hypertensive disorders of pregnancy. 
Pregnancy Hypertens 2014;4:105-45.

12. Santos VM, Correa FG, Modesto FR, Moutella PR. Late-onset postpartum eclampsia: still a diagnostic dilemma? Hong Kong Med J 2008;14:60-3.

13. Magee $L$, von Dadelszen P. Prevention and treatment of postpartum hypertension. Cochrane Database Syst Rev 2013;(4):CD004351.

14. Sibai BM. Etiology and management of postpartum hypertensionpreedlampsia. Am J Obstet Gynecol 2012;206:470-5.

15. Hinchey J, Chaves C, Appignani B, et al. A reversible posterior leukoencephalopathy syndrome. N Engl J Med 1996;334:494-500.

16. Fugate JE, Claassen DO, Cloft HJ, Kallmes DF, Kozak OS, Rabinstein AA. Posterior reversible encephalopathy syndrome: associated clinical and radiologic findings. Mayo Clin Proc 2010;85:427-32.

17. Narayan D, Kaul S, Ravishankar $K$, et al. Risk factors, clinical profile, and long-term outcome of 428 patients of cerebral sinus venous thrombosis: insights from Nizam's institute venous stroke registry, Hyderabad (India). Neurol India 2012;60:154-9.

18. Ferro JM, Canhão P, Stam J, Bousser MG, Barinagarrementeria F; ISCVT Investigators. Prognosis of cerebral vein and dural sinus thrombosis: results of the International Study on Cerebral Vein and Dural Sinus Thrombosis (ISCVT). Stroke 2004;35:664-70.

19. Lee VH, Wijdicks EF, Manno EM, Rabinstein AA. Clinical spectrum of reversible posterior leukoencephalopathy syndrome. Arch Neuro/ 2008; 65:205-10.
20. Shankar J, Banfield J. Posterior reversible encephalopathy syndrome: a review. Can Assoc Radiol J 2017;68:147-53.

21. Garg RK, Kumar N, Malhotra HS. Posterior reversible encephalopathy syndrome in eclampsia. Neurol India 2018;66:1316-23.

22. Roth $C$, Ferbert A. Posterior reversible encephalopathy syndrome: long-term follow-up. J Neurol Neurosurg Psychiatry 2010;81:773-7.

23. Goel A, Maski MR, Bajracharya S, et al. Epidemiology and mechanisms of de novo and persistent hypertension in the postpartum period. Circulation 2015;132:1726-33.

24. Kang E, Sugarman $R$, Ramadan $H$, et al. Prevalence, risk factors and associated complications of postpartum hypertension in rural Haiti. Pregnancy Hypertens 2017;10:135-42.

25. August P, Malha L. Postpartum hypertension: "It Ain't Over 'til It's Over". Circulation 2015;132:1690-2.

26. Liman TG, Bohner G, Heuschmann PU, Scheel M, Endres M, Siebert E. Clinical and radiological differences in posterior reversible encephalopathy syndrome between patients with preeclampsia-eclampsia and other predisposing diseases. Eur J Neurol 2012;19:935-43.

27. Danhofer $\mathrm{P}$, Tomečková $\mathrm{M}$, Černá, et al. Prognostic factors and seizure outcome in posterior reversible encephalopathy syndrome (PRES) in children with hematological malignancies and bone marrow failure: a retrospective monocentric study. Seizure 2019;72:1-10. 